\title{
Simulating the use of re-prioritisation as a wait-reduction strategy in an emergency department
}

\author{
Jane Coughlan, Julie Eatock, Nayna Patel
}

\section{ABSTRACT}

Background/aim Simulation modelling has proven a useful approach for captuning the dynamic nature of emergency departments (EDs) and informing improvements to clinical and operational processes alike. However, few models have simulated the impact of the UK Department of Health's $4 \mathrm{~h}$ operational standard, which arguably has placed pressure to improve standards and performance, promoting the use of wait-reduction strategies to cope with the target in practice. The aim of this study was to determine the impact a re-prioritisation strategy has on the $4 \mathrm{~h}$ target by simulating the operation of an ED using a model that represents the flow of patients through the department Methods This study was based on a district general hospital in West London. To ascertain patients' length of stay, the hospital's historical records and staff rotas were used to obtain data on activities, timeframes and resources on three separate representative weeks and included all patients' arrival time, mode of arrival, whether the patient was referred to minors, majors, paediatrics or the resuscitation unit, and whether the patient was admitted or discharged, and at what time. Results The close correlation (rr/40.98) in distributions between actual length of stay and simulated length of stay demonstrates that the model of the ED accurately replicates the $4 \mathrm{~h}$ peak caused by the use of re-prioritisation.

Conclusion The model accurately reproduced the use of a dominant wait-reduction strategy to identify patients approaching the breach and re-prioritise them to expedite treatment and remove them from the department by the $4 \mathrm{~h}$ target.

\section{INTRODUCTION}

In order to improve health services and the patients' experience, a number of governmentimposed targets have been introduced to address waiting times, notably in the context of emergency care. ${ }^{1}$ Since January 2005, emergency departments (EDs) in the UK have been required to ensure that at least $98 \%$ of patients are either discharged or admitted to hospital within $4 \mathrm{~h}$ of arrival. $^{2}$ However, target setting has increased the pressure on senior health service managers to perform, and consequently this has led to the use of waitreduction strategies. ${ }^{3}$ The types of strategies employed take the form of rationing and prioritisation, which are not uncommon management practices in the ED to reduce patient turnaround time. $^{4}$ However, the use of re-prioritisation (the practice of increasing a patient's priority and hence treating out of clinical order) in avoiding the breach of the $4 \mathrm{~h}$ target complicates yet further the dynamic and challenging environment of emergency care. ${ }^{5}$ Support for the $4 \mathrm{~h}$ target has been equivocal as, while there is some suggestion that care has improved in some areas, ${ }^{67}$ others cite the distortion of clinical priorities and compromised quality of care. ${ }^{8}$ The post-2010 elected government has announced it will scale back, amend and eventually relax the $4 \mathrm{~h}$ target, ${ }^{9}$ although at the time of writing the exact details of the reforms had not been set out, but it is likely to be replaced in some form where target-meeting strategies will develop accordingly.

Recent work has called for more research into informing the debate over the impact of time-driven targets on patient outcomes in EDs as evidence is scarce. ${ }^{10}$ Simulation studies that present models produced after the introduction of the $4 \mathrm{~h}$ operational standard are rarer still, ${ }^{11 e} 16$ and of these five studies, only two include it in their analysis of the patient throughput times. ${ }^{13}{ }^{16}$ Consequently, both conclude that emergency care staff quickly adapt their behaviour to meet the target, which has encouraged the development of wait-reduction strategies designed to cope with the imposed timeframe. Although these studies show that such strategies are being used in EDs and account for some discrepancies in their results, there appears to be no previous work that uses simulation to capture the wait-reduction strategies and their impact on the $4 \mathrm{~h}$ target. This is most clearly evidenced in a study by Gunal and Pidd ${ }^{13}$ who used simulation of an ED to understand the effects of waiting time targets in an ED. In their simulated performance of 'department $B^{\prime}$ they found a lack of agreement between the actual reported performance and the simulated performance (ie, the expected performance of a department running normally) around the $4 \mathrm{~h}$ mark. The substantial peak at $4 \mathrm{~h}$ in the hospital's reported data led the authors to suggest that the department is taking serious interventions to complete the processing of patients quickly as the deadline approaches.

Modelling an emergency care environment with an attempt to understand the impact of the interventions or wait-reduction strategies provides an opportunity to improve the performance of hospitals in attaining targets. However, Wolstenholme et $\mathrm{al}^{17}$ report on the difficulty in modelling the strategies given that they are to a greater extent informal and therefore unofficial. However, there is at least one example of a specific measure that has been taken with the introduction of a breach avoidance facilitator in an ED, which involves tasking senior nursing staff with a trouble-shooting role in managing the $4 \mathrm{~h}$ target. $^{18}$ Generally, however, there is no clear-cut understanding of the 
types of wait-reduction strategies used in emergency care, and research into identifying the range of practices employed and their impact is lacking. However, some examples of additional measures taken to reduce wait times are: gaming (data manipulation) strategies ${ }^{19}$; moving patients to medical assessment units and effectively discharging them ${ }^{20}$; and even bullying of nursing staff by senior management. ${ }^{21}$ The combined effect of re-prioritisation and these additional measures can be seen as a spike just before the $4 \mathrm{~h}$ breach occurs. ${ }^{22}$

Discrete event simulation is a modelling tool that can effectively capture interactions in complex processes as well as facilitating predictive analysis through the use of scenario modelling. The complexity of the patient journey, workflow and practices in emergency care, and their associated interactions, lends itself to this type of modelling. To increase the accuracy of the results obtained, the model needs to be a valid representation of actual activities within the ED. As the wait-reduction strategies constitute an integral part of emergency care, no model can be accurate without at least capturing some of the key strategies being used. Our study therefore aims to simulate the operation of an ED by building a simulation model that represents the flow of patients through the department and the impact that re-prioritisation has on the $4 \mathrm{~h}$ target.

\section{MODELLING AN ED}

This study is based on the ED of a district general hospital in West London. The trust has a single ED department as well as a satellite minor injuries unit. From the data supplied by the hospital the approximate percentage of patients sent to the four areas can be seen in table 1 .

In order to meet the $4 \mathrm{~h}$ target, this particular ED adopts a number of wait-reduction strategies (primarily the re-prioritisation of patients) and additional management practices (flexible staff breaks, deploying staff between different areas and expediting test results when necessary). In an attempt to represent some of these strategies within our model we used the hospital's historical records to obtain data on activities, timeframes and resources. The data were collected from three separate representative weeks (as determined by the hospital) and included all patients that were in, or arrived in, the department from Monday 00:00 through to Sunday 23:59. This included: the arrival time; mode of arrival (walk-in/ambulance); whether the patient was referred to minors, majors, paediatrics or the resuscitation unit; and whether the patient was ultimately admitted or discharged, and at what time. This information enabled us to understand patients' pathways through the department and ascertain patients' length of stay in the ED. Resources for the model were also obtained from hospital records, and in particular staff rotas that contained information about the number and availability of doctors and nurses.

This hospital has five distinct areas to which patients can be directed, which is dependent on: mode of arrival; age; and the severity of the complaint. Patients presenting with a non-urgent

\section{Table 1 Annual data}

\begin{tabular}{llll} 
Trust level* & \multicolumn{3}{c}{ Department level } \\
Total (approximate) & 100000 & Total (approximate) & 70000 \\
Age \#19 & $31 \%$ & Minors & $44.41 \%$ \\
Age $\$ 70$ & $12.7 \%$ & Majors & $30.51 \%$ \\
& & Paediatrics & $20.09 \%$ \\
& & Resus unit & $4.98 \%$
\end{tabular}

*Trust level data taken from HES (Hospital Episode Statistics) data. case may be directed to the resident general practitioner (GP), and children under 16 will be directed to paediatrics. Figure 1 provides an overview of the areas in the ED.

A model of the ED was built using Simul8 Professional Edition version 15.0. The entire model is too large and complex to display here so a simplified view of the minors area is shown for illustrative purposes (figure 2), where the various activities and queues can be clearly seen. The other areas within the ED are modelled at a similar level of detail.

\section{MODELLING THE RE-PRIORITISATION STRATEGY}

Re-prioritisation is the most dominant wait-reduction strategy adopted within this ED. The concept underpinning this strategy is constantly to review and escalate a patient as they work their way through the ED, with the aim of being discharged in advance of reaching the $4 \mathrm{~h}$ target. This strategy uses patient priority ratings and updates them at distinct times throughout their stay in the department. The following provides a more detailed description of the re-prioritisation strategy and how it was represented within our simulation model.

\section{Patient arrival and referral}

When a patient enters the ED the model records their arrival time. Patients that arrive by ambulance are, in the majority of cases, allocated with the highest priority and handled within the model by fast-tracking them directly to the majors area or the resuscitation unit. After reporting to Reception, walk-in patients are promptly triaged by a nurse, and assigned a priority rating based on the severity of their case. Patients are then referred to the relevant area within the ED (minors, majors, paediatrics or the resuscitation unit). Within the model, the allocation of patients to specific areas is based on the data obtained from the hospital.

As they move through the model, their priority rating is reviewed and updated at set intervals $(150,180,210,230$ and $240 \mathrm{~min}$ ) based on the time that they arrived in ED. This is done on an individual patient basis and therefore the time until the next update is calculated each time a patient joins a queue. Figure 3 shows how the assignment and update of the priority ratings is achieved.

\section{Assessment, treatment and discharge}

Once a patient has been referred to the appropriate department, they queue in that particular area (majors, minors, etc.) to be seen by a member of the medical team. As a patient joins a queue, those with the highest priority rating are placed at the front of each queue. The model will only release a patient from any queue if the relevant resources to complete that task are available, for example on the condition that both a nurse and a cubicle are free.

In a real ED, once a patient is assessed they may receive a selection of diagnostic tests and/or treatments depending on their case. However, due to the extensive range of tests and treatments available the model would become overly complex if every individual test and different types of treatment were included. Therefore, this was overcome by having a single activity to represent the diagnostic tests and another to represent treatments. Statistical distributions were used to represent the likelihood of receiving a test and/or treatment as well as to provide a range of times to represent the variation of tests and treatments provided. If a patient from the minors area requires a test outside of the ED, for example an x-ray, the model releases the cubicle ready for the next patient in the queue. On their return, they are required to rejoin the appropriate queue and 
Figure 1 Oveniew of emergency department (ED). GP, general practitioner.

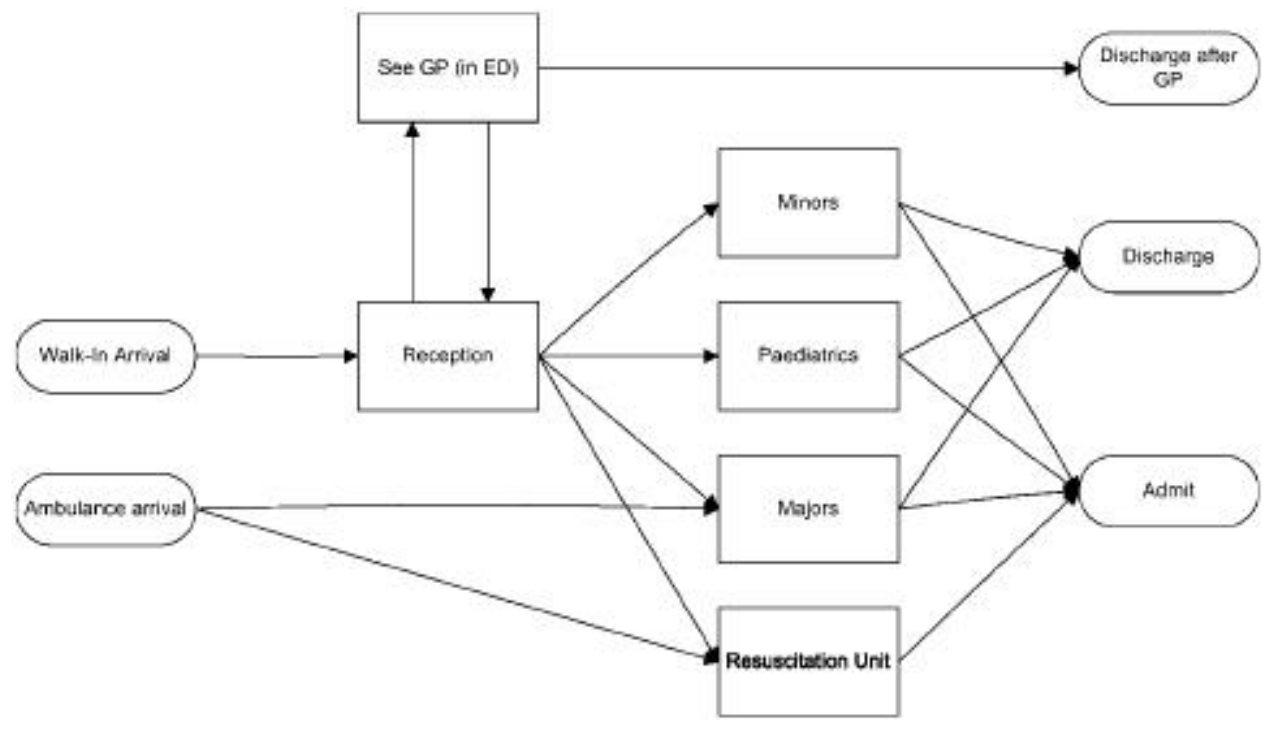

wait for the relevant resources to become available before they can continue their treatment.

In cases where a patient is approaching breach, the re-prioritisation strategy includes fast-tracking patients through the process. Figure 4 provides an overview of how this is applied within the model. Once the patient has been re-assessed after tests and/or treatment, they are either discharged, or admitted and the time they have spent in the department is calculated.

\section{ADDITIONAL MANAGEMENT PRACTICES}

In addition to re-prioritisation, this hospital implements a number of other management practices within their ED to reduce waiting times while ensuring that the sickest patients are treated expediently. Some of these practices are captured within the model, some approximated, while others have not been included because they fall outside the scope of the model or their frequency is negligible. A practice that was modelled is where staff are called in from the other areas of the ED to the resuscitation unit and then released back to their respective areas once their assistance is no longer required. Though this practice is intended to provide prompt treatment to the sickest patients, it has a large impact on the time other patients spend in the ED and therefore was included within this model. An example of a practice that is approximated by the model is the use of a flexible break system where, in reality, staff members take their breaks during the quieter periods, rather than following a strict timetable of scheduled breaks. This approach proved difficult to replicate within our model without overutilising the staff while ensuring ample resources were available at any time. The most appropriate option was to incorporate scheduled breaks within the various shift patterns. This, however, means that in the model staff may leave the department for a break during busy periods rather than waiting for a quieter time, which may produce slightly more pessimistic results than in reality. A practice that was not explicitly captured is contacting the

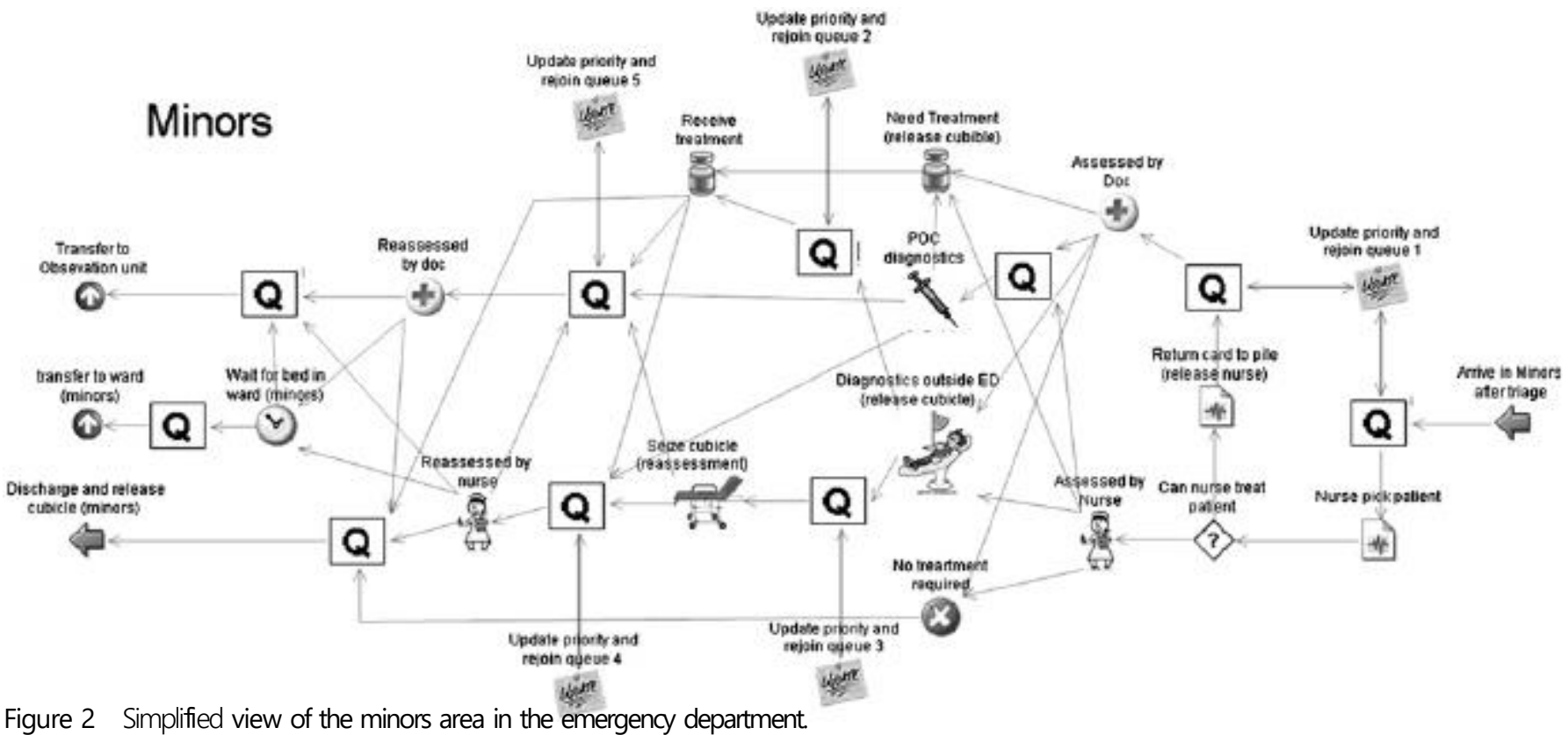

Figure 2 Simplified view of the minors area in the emergency department. 


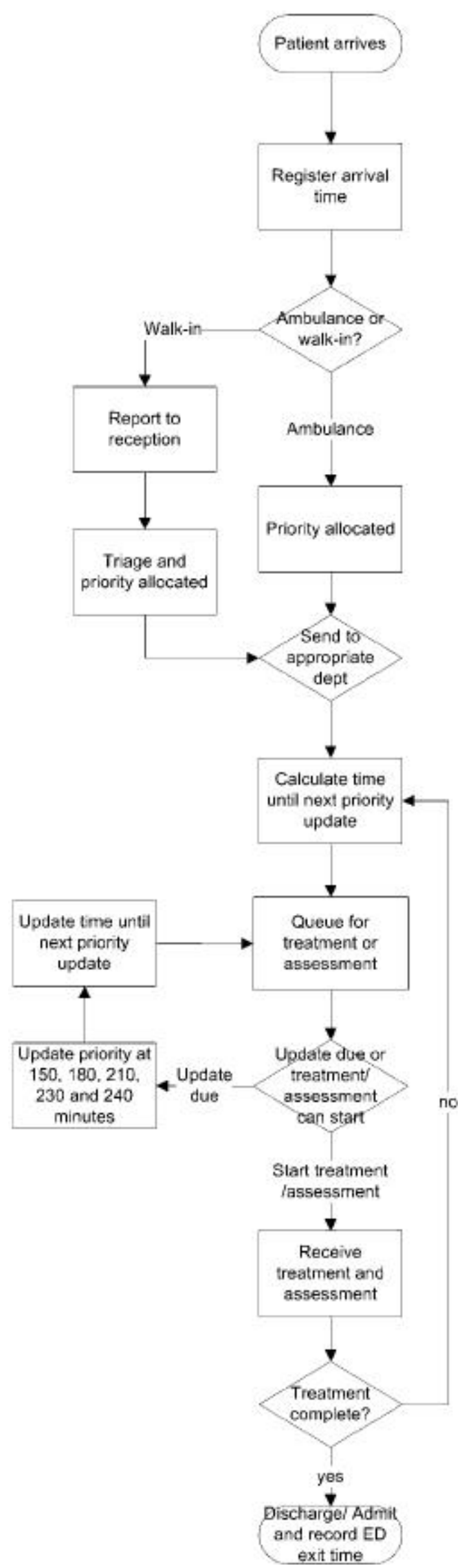

Figure 3 Priority rating flowchart. ED, emergency department.

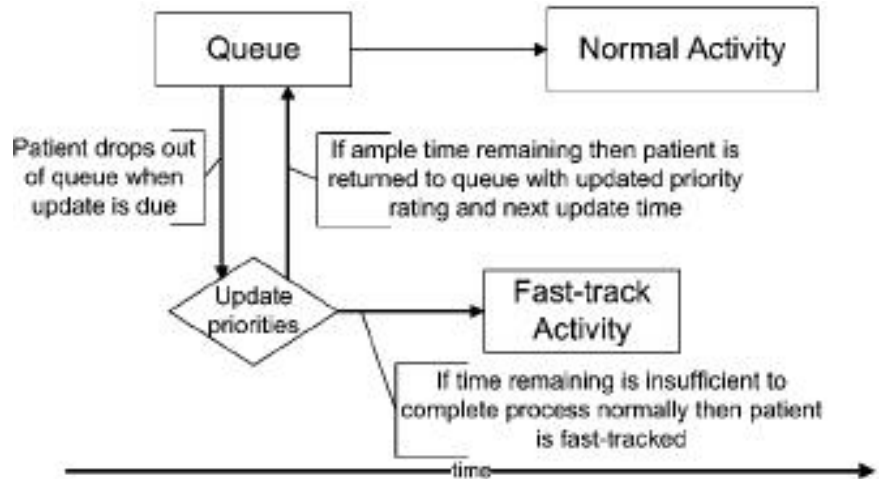

Figure 4 Fast tracking flowchart.

pathology laboratory to expedite the delivery of diagnostic test results when a patient is in danger of breaching the $4 \mathrm{~h}$ target. Activities within the pathology laboratory lie outside the control of the ED, and therefore outside the scope of this particular model. In making decisions regarding the practices that can be, and should be included within the model, we were required to make trade-offs between incorporating all the additional practices, which would make the model more complex, and the additional level of accuracy that could be achieved by their inclusion.

\section{RESULTS AND DISCUSSION}

In order to determine how well we have captured the effects of the wait-reduction strategies within the model we compared the actual length of stay with the length of stay times generated by the model. We took the hospital data for the week beginning 14 April 2008, and used the actual arrival data to populate the model.

From the graph shown in figure 5 , it can be seen that the model produces a similar distribution for the simulated length of stay to the actual length of stay, and accurately replicates the $4 \mathrm{~h}$ peak caused by the use of the re-prioritisation strategy. There is a slight discrepancy with the number of patients released between 240 and $270 \mathrm{~min}$, which in turn means that the percentage of patients through ED in $<4 \mathrm{~h}$ is slightly smaller than recorded in the hospital's actual figures. One reason for this could be the digit bias as reported by Locker and Mason, ${ }^{19}$ where patients released at, for example, 4:01 may have their time recorded as 3:59 or rounded to the nearest $5 \mathrm{~min}$ so as not to count as a breach case. We elected not to replicate this digit bias in the model as the aim is to improve throughput and so reduce the need for any data manipulation. Including the digit bias

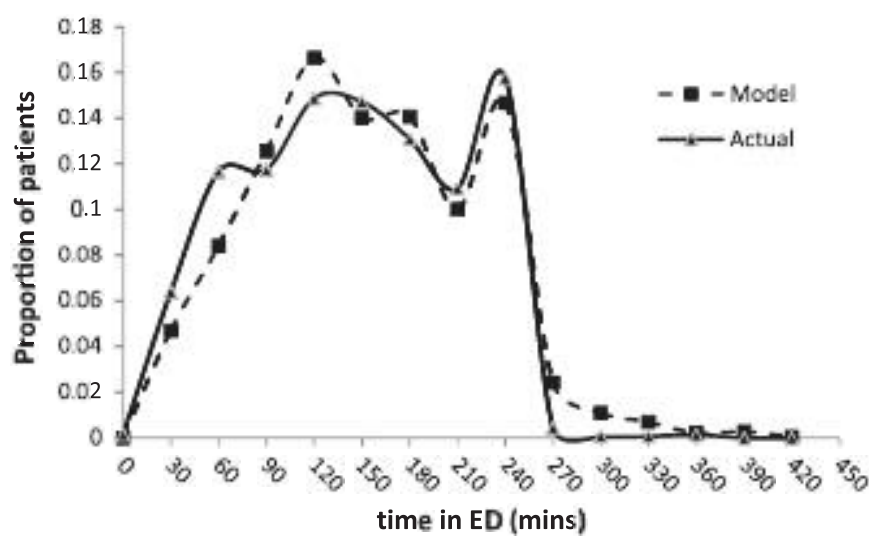

Figure 5 Comparison of length of stay in emergency department (ED). 
within the model may well distort the effects of changes made to the system. The model was also run for two other weeks, (the weeks beginning 27 July and 3 August 2008), and the results compared with the actual times that patients spent in the department during these weeks. The close correlation $\left(r^{1} / 40.98\right)$ between actual and predicted gives confidence in our model to provide insight into behaviour within the ED. It also illustrates that there is no need to increase the complexity of the model by adding additional wait-reduction strategies, as the improvement in output would be minimal.

Our initial work, as exemplified in figure 5, gave us a high level of confidence in the model and its ability to reproduce the characteristics of the actual length of stay distribution. Specifically, we have been able to reproduce some of the practices used within the ED to identify patients approaching the breach and re-prioritise them to expedite treatment and remove them from the department by the $4 \mathrm{~h}$ target. One of the main advantages of developing this type of model is the insight that can be acquired by analysis of the various processes and their impact. For instance, waiting for blood test results can account for a large percentage of a patient's time in ED, which could possibly be reduced by using point-of-care devices, at least for some patients. This in turn may free up cubicles earlier, allowing subsequent patients to be seen more quickly, reducing the need for wait-reduction strategies to be implemented.

In light of the accuracy of this model, we were able to use it as a basis to examine two further scenarios. The model of the first scenario demonstrated that using an Emergency Nurse Practitioner (also known as 'see and treat') to deal with the most minor injuries did not improve the times taken to treat patients. The second scenario involved the reallocation of resources to optimise their usage and resulted in improved throughput times. The accuracy of the baseline model provides us with confidence that the results obtained from modelling these scenarios would be borne out if implemented. Using the model in this way will allow selection of optimal strategies without the cost, time and disruption caused by actual implementation of ineffective strategies.

\section{FUTURE WORK}

Within our study we were able to represent accurately the impact of wait-reduction strategies within an ED using simulation. However, one of the criticisms of simulation models is that they are generally designed for a specific hospital and are not generalisable enough to be used in other hospitals. In this instance, the ability to produce a generic model that is flexible enough to allow adaptation for different scenarios and intuitive enough for hospital managers to use is important. ${ }^{23}$ Allowing hospital managers to input their own data on patient admissions, staff rotas, resources and management practices into a generic model would make this a cheaper and quicker way of developing a model that was representative of their particular hospital. The additional benefits of a generic model would be that it would facilitate the sharing of best practice, as well as successful (and unsuccessful) improvement strategies. Therefore, the authors' future work involves determining whether a level of homogeneity exists between different hospitals, and if so, to what extent, and whether this can be used as a basis for a generic model.

The HES (Hospital Episode Statistics) data ${ }^{22}$ show that three distinct patient discharge patterns exist within the UK, and this may equate to three different 'types' of EDs being in existence. If EDs can be categorised in this way this may provide a basis for the development of such a set of generic models capable of representing all, or a subset of, hospitals and may have implications for policy implementation. With regards to the contribution of this particular study, a recent survey by Fletcher and Worthington $^{24}$ of healthcare models surmised that the more complex a model is, the more specific, and therefore less generalisable, it becomes. The complexity of our model would therefore imply that it was unsuitable for the basis of a generic model. However, the complexities in this model lie in the re-prioritising, and fast-tracking of patients, which are management practices in use by many hospitals. ${ }^{21}$ The activities themselves that make up much of the model, such as diagnostic tests and treatments, are indeed at a very generic level and therefore could form the basis of a general model. Future study is required to determine whether all the processes surrounding these activities are also at a generic enough level to represent the way other hospitals operate. The authors maintain that the way this model has been designed would allow for rapid re-development into a generic model.

Funding JE acknowledges support of this work through the MATCH (Multidisciplinary Assessment of Technology Centre for Healthcare) Programme (EPSRC Grant GR/S29874/01), although the views expressed are entirely those of the authors.

\section{REFERENCES}

1. Commission for Healthcare Audit and Inspection. Accident and emergency. 2005. http://www.cqc.org.uk/_db/_documents/04019296.pdf

2. Department of Health. Reforming emergency care: first steps to a new approach. 2001. http://www.dh.gov.uk/en/Publicationsandstatistics/Publications/ PublicationsPolicyAndGuidance/DH_4008702

3. Kreindler S. Policy strategies to reduce waits for elective care: a synthesis of intemational evidence. Br Med Bull. Published Online First: 10 May 2010. doi:10.1093/bmb/ldq014

4. Cross J. Rationing in the emergency department the good, the bad, and the unacceptable. Emerg Med J 2005;22:171 e6.

5. Gubb J. Have targets done more harm than good in the English NHS? Br Med J 2009;338:a3130.

6. Mortimer A, Cooper S. The 4-hour target emergency nurses' views. Emerg Med J 2007;24:402e4.

7. Bevan G. Have targets done more ham than good in the English NHS? Br Med J 2009;338:a3129.

8. Spicer R. Targets ham patients and are destroying the English NHS. Int J Clin Pract 2009;63:1278e81.

9. Kmietowicz Z. Four hour targets for accident and emergency are to be scrapped. Br Med J 2010;340:c3122.

10. Freeman J, Croft S, Cross S, et al. The impact of the $4 \mathrm{~h}$ target on patient care and outcomes in the Emergency Department an analysis of hospital incidence data. Emerg Med J. Published Online First: 13 May 2010. doi:10.1136/ emj.2009.085431.

11. Codrington-Virtue $A$, Chaussalet $T$, Millard $P$, et al. A system for patient management based discrete-event simulation and hierarchical clustering. In: Proceedings of the IEEE Symposium on Computer-Based Medical Systems. Salt Lake City, Utah, 2006:800e4.

12. Gunal M, Pidd M. Understanding accident and emergency department performance using simulation. In: Proceedings of the Winter Simulation Conference. Monterey, California, 2006:446e52.

13. Gunal M, Pidd M. Understanding target-driven action in emergency department performance using simulation. Emerg Med J 2009;26:724e7.

14. Davies R. "See and Treat" or "See" and "Treat" in an Emergency Department In: Proceedings of the Winter Simulation Conference. Washington, DC, 2007: 1519e22.

15. Bowers J, Ghattas M, Mould G. Success and failure in the simulation of an Accident and Emergency department J Simul 2009;3:171e8.

16. Maulla $R$, Smarta $P$, Hamis $A$, et al. An evaluation of 'fast track' in A\&E: a discrete event simulation approach. Serv Ind J 2009;29:923 e41.

17. Wolstenholme E, Monk D, McKelvie D, et al. Coping but not coping in health and social care: masking the reality of running organisations beyond safe design capacity. Syst Dynam Rev 2007;23:371e89.

18. Cronin J, Wright J. Breach avoidance facilitatord managing the A\&E 4-hour target. Accid Emerg Nurs 2006;14:43e8.

19. Locker T, Mason S. Are these emergency department performance data real? Emerg Med J 2006;23:558e9.

20. Mayhew L, Smith D. Using queuing theory to analyse the Govemment's 4-h completion target in accident and emergency departments. Healthcare Manag Sci 2008;11:11e21. 
21. BMA. Emergency medicine: report of national survey of emergency medicine. 2007. http://www.bma.org.uk/images/Emergencymedicine_tcm41-146692.pdf

22. HES Online. Accident and emergency d patient joumey. further analysis of the published 2007e08 A\&E HES data (experimental statistics). http://www.ic.nhs.uk/ webfiles/publications/hes/AandE/Accident_and_emergency_attendances_in_ England_2007_08.pdf (accessed 3 Nov 2010).

23. Sinreich $D$, Marmor $Y$. A simple and intuitive simulation tool for analyzing emergency department operations. In: Proceedings of the Winter Simulation Conference, Washington, D.C, 2004:1994e2002.

24. Fletcher A, Worthington D. What is a 'Generic' hospital model? $\mathbf{d}$ a comparison of 'generic' and 'specific' hospital models of emergency patient flows. Healthcare Manag Sci 2009;12:374e91. 